\title{
ROBUST LOW-COMPLEXITY MULTICHANNEL EQUALIZATION FOR DEREVERBERATION
}

\author{
Felicia Lim and Patrick A. Naylor \\ Dept. of Electrical and Electronic Engineering, Imperial College London, UK \\ \{felicia.lim06, p.naylor\}eimperial.ac.uk
}

\begin{abstract}
Multichannel equalization of acoustic impulse responses (AIRs) is an important approach for dereverberation. Since AIRs are inevitably estimated with system identification error (SIE), it is necessary to develop equalization designs that are robust to such SIE, in order for dereverberation processing to be beneficial. We present here a novel subband equalizer employing the relaxed multichannel least squares (RMCLS) algorithm in each subband. We show that this new structure brings improved performance in dereverberation as well as a reduction in computational load by up to a factor of more than 90 in our experiments. We then develop a novel controller for the dereverberation processing in subbands that guarantees robustness to even very severe SIEs by backing off dereverberation in any subband with excessively high levels of SIEs.
\end{abstract}

Index Terms - Dereverberation, equalization, computational complexity, robustness, system identification errors.

\section{INTRODUCTION}

In hands-free communication, the signal captured at the microphone(s) typically suffer from reverberation as a result of the propagation of the source signal through a multipath acoustic channel, which can be modeled by an acoustic impulse response (AIR). Reverberation can negatively affect the perceived speech quality and the performance of speech recognizers [1].

A promising approach to dereverberation for multichannel systems is to estimate the AIRs using blind system identification algorithms such as $[2,3,4]$ and then apply inverse filters for the AIRs in order to recover an estimate of the original (dry) signal.

An exact multichannel inverse filter can be calculated using the multiple-input/output inverse theorem (MINT) algorithm [5], subject to the conditions given in Section 2. However, in the presence of system identification errors (SIEs), the exact inverse filters provided by MINT do not equalize the true AIRs and the overall effect is actually to add reverberation rather than suppress it as desired. In [6], regularization was introduced to increase the robustness of MINT. The relaxed multichannel least squares (RMCLS) [7], in contrast, relaxes constraints on the equalizer design to increase robustness to SIEs in a manner desirable for dereverberation. It is an efficient implementation of the channel shortening paradigm, which has been well developed in digital communications $[8,9,10,11]$ and recently studied for acoustic applications $[12,13,14]$ to exploit the psychoacoustics principle that early reflections in an AIR corresponding to approximately the first $0.05 \mathrm{~s}$ have been shown to contribute positively to speech intelligibility [1]. On the other hand, the late reflections contribute to the degradation of perceived speech quality [1] and it is therefore desirable to suppress late reverberant coefficients in the equalized impulse response (EIR). While RMCLS provides greatly improved robustness over MINT in moderate levels of SIEs, its performance is limited in very high levels of SIEs and it cannot be guaranteed that the output is uncoloured. In [15], we proposed constraint of initial taps to control the levels of colouration introduced. We now propose a novel algorithm, gated subband multichannel equalization and demonstrate that it offers three benefits over existing algorithms. The first benefit is an improvement in the reverberant tail suppression in the EIR. The second benefit is robustness so as to limit the degradation introduced in the EIR in the presence of very high levels of SIEs. The third benefit is a significant reduction in computational complexity with a reduction in runtime.

Relation to prior work - We implement RMCLS in subbands (RMCLS-SB) and propose gated subband equalization to exploit the flexibility, increased robustness and reduced computational complexity afforded by subband processing. This provides improved performance in the presence of severe SIEs over full-band equalization algorithms MINT [5] and RMCLS [7], and extends the work in [16] where only MINT in subbands was considered.

\section{REVIEW OF MULTICHANNEL EQUALIZATION}

An $M$-channel $(M \geq 2)$ acoustic system can be characterized by $M$ finite impulse response (FIR) impulse responses, $\mathbf{h}_{m}=$ $\left[h_{m}(0) h_{m}(1) \ldots h_{m}(L-1)\right]^{T}$ for $m=1,2, \ldots, M$. A speech signal $s(n)$ propagating through this acoustic system can then be described at the $m$-th microphone by

$$
\mathbf{x}_{m}(n)=\mathbf{H}_{m}^{T} \mathbf{s}(n)+\mathbf{v}(n)
$$

where $\mathbf{x}_{m}(n)=\left[x_{m}(n) x_{m}(n-1) \ldots x_{m}\left(n-L_{i}+1\right)\right]^{T}, \mathbf{s}(n)=$ $\left[s(n) s(n-1) \ldots s\left(n-L-L_{i}+2\right)\right]^{T}$ and $\mathbf{v}(n)=[v(n) v(n-$ 1) $\left.\ldots v\left(n-L_{i}+1\right)\right]^{T}$ denote segments of the microphone, speech and noise signals respectively, $\mathbf{H}_{m}$ is the $\left(L+L_{i}-1\right) \times L_{i}$ convolution matrix of $\mathbf{h}_{m}$ and $L_{i}$ is the equalizing filter length.

The reverberant speech signals $\mathbf{x}_{m}(n)$ can be equalized using filters $\mathbf{g}_{m}=\left[\begin{array}{llll}g_{m}(0) & g_{m}(1) & \ldots & g_{m}\left(L_{i}-1\right)\end{array}\right]^{T}$ which invert the effect of $\mathbf{h}_{m}$ to give an EIR $\mathbf{r}$ such that $\mathbf{H g}=\mathbf{r}$, where $\mathbf{H}=\left[\begin{array}{lllll}\mathbf{H}_{1} & \mathbf{H}_{2} & \ldots & \mathbf{H}_{M}\end{array}\right], \mathbf{g}=\left[\begin{array}{llll}\mathbf{g}_{1}^{T} & \mathbf{g}_{2}^{T} & \ldots & \mathbf{g}_{M}^{T}\end{array}\right]^{T}$ and $\mathbf{r}=\left[r(0) r(1) \ldots r\left(L+L_{i}-2\right)\right]^{T}$.

The problem addressed is now formulated as follows. Given estimated AIRs $\hat{\mathbf{h}}=\mathbf{h}+\mathbf{e}$, where $\mathbf{h}=\left[\begin{array}{llll}\mathbf{h}_{1} \mathbf{h}_{2} & \ldots \mathbf{h}_{M}\end{array}\right]$ and $\mathbf{e}$ are SIEs quantified by normalized projection misalignment (NPM) [4], find a set of filters $\mathbf{g}$ which equalize the true $\mathbf{h}$ in a robust manner.

\subsection{Multiple-input/output Inverse Theorem (MINT)}

MINT [5] provides exact inverse filters $\mathbf{g}$ by selecting the desired EIR to be $\mathbf{r}=\mathbf{d}$, where $\mathbf{d}=\left[\begin{array}{llll}1 & 0 & \ldots & 0\end{array}\right]_{\left(L+L_{i}-1\right) \times 1}^{T}$. A solution to $\mathrm{g}$ is given by

$$
\mathbf{g}=\hat{\mathbf{H}}^{+} \mathbf{d}
$$




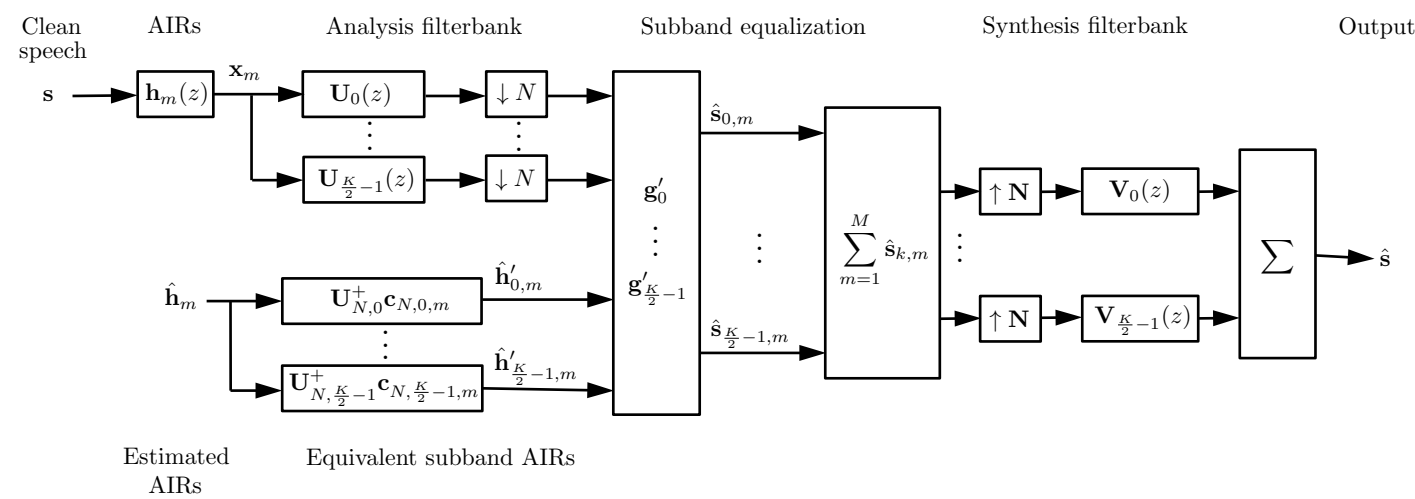

Fig. 1. Structure of multichannel equalization in subbands.

where $\hat{\mathbf{H}}$ is formed from $\hat{\mathbf{h}}$ and $\{\cdot\}^{+}$denotes the Moore-Penrose pseudo-inverse, and exists subject to $M \geq 2$ and the following conditions [5, 17]

C1 $H_{m}(z)$, the z-transforms of $\mathbf{h}_{m}$, do not share any common zero.

C2 $L_{i} \geq\left\lceil\frac{L-1}{M-1}\right\rceil$, where $\lceil\cdot\rceil$ is the ceiling operator.

While MINT is able to provide exact inverses of multichannel AIRs, it is not robust to the presence of SIEs.

\subsection{Relaxed Multichannel Least Squares (RMCLS)}

RMCLS [7] improves the robustness of an equalizer design to SIEs by relaxing the constraints on the early coefficients of $\mathbf{r}$. This is achieved by minimizing the following cost function

$$
J=\left\|\mathbf{W}_{r}(\hat{\mathbf{H} g}-\mathbf{d})\right\|_{2}^{2},
$$

where $\mathbf{W}_{r}=\operatorname{diag}\left\{\mathbf{w}_{r}\right\}$ with $\mathbf{w}_{r}$ given by

$$
\mathbf{w}_{r}=[\underbrace{1 \ldots 1}_{\tau} \underbrace{10 \ldots 0}_{L_{w}} 1 \ldots 1]_{\left(L+L_{i}-1\right) \times 1}^{T} .
$$

The term $\tau$ denotes delay of the direct path in the AIR and $L_{w}$ defines the length of the relaxation window corresponding to the region of early reflections of an AIR. The first weight in the relaxation window is set to 1 to avoid the trivial solution. The minimum $\ell_{2}$-norm solution can be obtained from

$$
\mathbf{g}=\left(\mathbf{W}_{r} \hat{\mathbf{H}}\right)^{+} \mathbf{W}_{r} \mathbf{d} .
$$

Although RMCLS provides improved robustness to SIEs compared to MINT due to the relaxation of constraints, its performance is limited in the presence of very large SIEs. Additionally, both MINT and RMCLS require matrix inverses, which are computationally intensive. This limits their usefulness in practical implementations.

\section{GATED SUBBAND MULTICHANNEL EQUALIZATION}

We propose a subband equalizing structure, gated RMCLS-SB (RMCLS-SB-G), which simultaneously addresses both issues of robustness and computational complexity associated with RMCLS. We include gated equalization employing RMCLS in each subband where equalization is applied only if the expected NPM of the subband SIE, $\tilde{e}_{k}$, is better than a threshold $\mathrm{NPM}_{\mathrm{t}}$, determined by the level of SIEs to which the equalization algorithm is no longer robust. This enables a limitation to be placed so as to avoid any additional degradation introduced in $\mathbf{r}$ by errant equalizer design in the case of very high levels of SIEs. To find $\mathrm{NPM}_{t}$, non-robustness is defined here as the case where the energy in the reverberant tail of $\mathbf{r}$ is greater than the energy in the reverberant tail of $\mathbf{h}$. The energy in the EIR is quantified using energy decay curves (EDCs) [1] and the region of reverberant tail is defined as $t>0.05 \mathrm{~s}$ [1]. In this work, the oracle case is assumed where exact SIEs, $e_{k}$, are known. In practical applications, $\tilde{e}_{k}$ could be estimated from signal-to-noise ratio (SNR) estimates $[18,19]$. The framework for implementing RMCLS-SB is next presented.

\subsection{Subband Implementation}

Early investigations into multichannel acoustic equalization in subbands [20] employed the use of MINT in critically decimated filterbanks, where estimations of the equivalent subband AIRs were performed using the method of least squares based on the microphone signals. In [21], the relationship between filtering in full-band and subband was investigated and it was shown that a diagonal filtering matrix can be introduced such that performing convolution in subbands give good approximations to performing convolution in full-band. This approach requires the implementation of only one equalizing filter in each subband, and was used in [16] for the multichannel case in conjunction with oversampled subbands. We now follow a corresponding approach for implementing RMCLS in subbands, detailed next with reference to the system diagram in Fig. 1.

We employ the generalized discrete Fourier transform (GDFT) filter-bank [22] for the analysis and synthesis filters. For a $K$ subband system, the analysis filters $u_{k}(n), k=0, \ldots, K$ are calculated by modulating a prototype filter $p(n)$ such that

$$
u_{k}(n)=p(n) \cdot e^{j \frac{2 \pi}{K}\left(k+k_{0}\right)\left(n+n_{0}\right)}
$$

where $k_{0}$ and $n_{0}$ are frequency and time offset terms, set to $k_{0}=$ $1 / 2$ and $n_{0}=0$ as in [21, 16]. Synthesis filters satisfying near perfect reconstruction are given by the time-reversed and conjugated analysis filters [21], $v_{k}(n)=u_{k}^{*}\left(L_{\mathrm{pr}}-n-1\right)$, where $L_{\mathrm{pr}}$ denotes the length of the prototype filter and is therefore also the length of the analysis and synthesis filters. As in [16], $K=32, N=24$ and an $L_{\mathrm{pr}}=512$-tap prototype filter were chosen as an illustrative example with good trade-offs between sufficient suppression of aliasing and sufficiently short subband equalization filters. With this filter design, processing of only the first $K / 2$ subbands is required since for real input signals, the remaining $K / 2$ subbands are simply complex conjugates of the first $K / 2$ subbands [21]. 
To design RMCLS-SB equalizing filters $\mathbf{g}^{\prime}$, the equivalent subband estimated AIRs $\hat{\mathbf{h}}_{k m}^{\prime}$ must first be found from the full-band version $\hat{\mathbf{h}}_{m}$. Complex subband decomposition $[16,21]$ can be employed to find $\hat{\mathbf{h}}_{k m}^{\prime}$, given by

$$
\hat{\mathbf{h}}_{k m}^{\prime}=\mathbf{U}_{N, k}^{+} \mathbf{c}_{N, k m}
$$

where

$$
\mathbf{U}_{N, k}=\left[\begin{array}{cccc}
u_{k}(0) & 0 & \cdots & 0 \\
u_{k}(N) & u_{k}(0) & \cdots & 0 \\
\vdots & \ddots & \ddots & \vdots \\
u_{k}\left(L_{\mathrm{pr}}-1\right) & \ldots & \vdots & 0 \\
0 & u_{k}\left(L_{\mathrm{pr}}-1\right) & \ddots & \vdots \\
\vdots & \vdots & \ddots & \vdots \\
0 & \cdots & 0 & u_{k}\left(L_{\mathrm{pr}}-1\right)
\end{array}\right]
$$

and $\mathbf{c}_{N, k m}=\left[\begin{array}{llll}c_{k m}(0) & c_{k m}(N) & \ldots & c_{k m}(N(L-1))\end{array}\right]^{T}$ is an $\left\lceil\left(L+L_{\mathrm{pr}}-1\right) / N\right\rceil \times 1$ vector with $c_{k m}(n)=\hat{h}_{m}(n) * u_{k}(n)$. The resulting length of $\hat{\mathbf{h}}_{k m}^{\prime}$ is $L^{\prime}=\left\lceil\frac{L+L_{\mathrm{pr}}-1}{N}\right\rceil-\left\lceil\frac{L_{\mathrm{pr}}}{N}\right\rceil+1$ [21].

\subsection{Multichannel Equalization in Subbands}

From $\hat{\mathbf{h}}_{k m}^{\prime}$, the equivalent subband $\hat{\mathbf{H}}$, denoted by $\hat{\mathbf{H}}_{k}^{\prime}$, can be found so as to enable the design of subband equalizing filters. It is known [16] that the solution for MINT-subband (MINT-SB) can be found by modifying (2) to give

$$
\mathbf{g}_{k}^{\prime}=\hat{\mathbf{H}}_{k}^{\prime+} \mathbf{d}^{\prime} \quad \text { for } k=0,1, \ldots, K / 2-1,
$$

where $\mathbf{d}^{\prime}=\left[\begin{array}{llll}1 & 0 & \ldots & 0\end{array}\right]_{\left(L^{\prime}+L_{i}^{\prime}-1\right) \times 1}^{T}$ and $L_{i}^{\prime}=\left\lceil\frac{L^{\prime}-1}{M-1}\right\rceil$.

Equivalently, the solution for RMCLS-SB can be found by modifying (5) to give

$$
\mathbf{g}_{k}^{\prime}=\left(\mathbf{W}_{r}^{\prime} \hat{\mathbf{H}}_{k}^{\prime}\right)^{+} \mathbf{W}_{r}^{\prime} \mathbf{d}^{\prime} \quad \text { for } k=0,1, \ldots, K / 2-1,
$$

where $\mathbf{W}_{r}^{\prime}=\operatorname{diag}\left\{\mathbf{w}_{r}^{\prime}\right\}$ with

$$
\mathbf{w}_{r}^{\prime}=[\underbrace{1 \ldots 1}_{\tau^{\prime}} \underbrace{10 \ldots 0}_{L_{w}^{\prime}} 1 \ldots 1]_{\left(L^{\prime}+L_{i}^{\prime}-1\right) \times 1}^{T},
$$

where $\tau^{\prime}=\lceil\tau / N\rceil, L_{w}^{\prime}=\left\lceil L_{w} /(\alpha N)\right\rceil$ and $\alpha=4 / 3$ was used as an illustrative example to give good overall results. A detailed study of the choice of $\alpha$ remains as future research.

The subband reverberant signals, $x_{k m}(n)=x_{m}(n) * u_{k}(n)$, can then be equalized with $\mathbf{g}_{k}^{\prime}$ and reconstructed to give the full-band equalized signal $\hat{\mathrm{s}}$ as in Fig. 1.

\section{COMPUTATIONAL CONSIDERATIONS}

Both MINT and RMCLS require computationally intensive pseudoinverses of large matrices, $\mathbf{A}^{+}$. Additionally, the matrix for RMCLS is normally very ill-conditioned due to the relaxed window. Subband processing provides improvements in both respects.

To gain insight, the condition numbers for the full-band and subband RMCLS matrices, $\mathbf{W}_{r} \hat{\mathbf{H}}$ and $\mathbf{W}_{r}^{\prime} \hat{\mathbf{H}}_{k}^{\prime}$, were computed for 50 different $\hat{\mathbf{H}}$ based on the same acoustic system with varying sourcemicrophone configuration to give spatially averaged results. The average condition number for RMCLS is $6.0 \times 10^{19}$ with a variance of $7.7 \times 10^{39}$. In contrast, the average condition number for RMCLS-SB is $3.1 \times 10^{17}$ with a variance of $1.7 \times 10^{33}$.

The computational load in RMCLS is dominated by calculating $\mathbf{A}^{+}$, which can be found using, for example, MATLAB's pinv function. This implements Golub-Reinsch SVD [23] which has a computational complexity of $4 y z^{2}+8 z^{3}$ flops [24], where $y$ and $z$ are the number of rows and columns in $\mathbf{A}$ respectively and a flop is either one real multiplication or addition. For the full-band algorithms, the matrix size is given by $y_{f b}=\left(L+L_{i}-1\right)$ and $z_{f b}=M L_{i}$. For the subband algorithms, $y_{s b}=\left(L^{\prime}+L_{i}^{\prime}-1\right)$ and $z_{s b}=M L_{i}^{\prime}$. The complex decomposition filters $\mathbf{U}_{N, k}^{+}$in (7) should also be considered, where its size is given by $y_{u}=\left\lceil\left(L+L_{\mathrm{pr}}-1\right) / N\right\rceil$ and $z_{u}=L^{\prime}$. For subband algorithms, a factor of $4 K / 2$ is applied to reflect the $K / 2$ number of subbands processed and the factor 4 accounts for 4 real multiplies per complex multiply in the complex filter bank employed. The reduction in computational complexity associated with the subband structure is

$$
\frac{4 y_{f b} z_{f b}^{2}+8 z_{f b}^{3}}{2 K\left(4 y_{s b} z_{s b}^{2}+8 z_{s b}^{3}+4 y_{u} z_{u}^{2}+8 z_{u}^{3}\right)} .
$$

In this work for example, using $K=32, N=24, L_{\mathrm{pr}}=512$ and an acoustic system with $L=2000$ and $M=2$, the reduction factor achieved is 191 . Reductions in runtime are additionally measured by MATLAB's cputime function and provided in Section 5 .

\section{SIMULATIONS AND RESULTS}

Two simulations were conducted to evaluate the performances of RMCLS-SB and RMCLS-SB-G in the presence of SIEs. Evaluation was conducted with three measures. The first measure evaluates the reverberant tail suppression achieved in the EIR, obtained using EDCs. The second measures the perceived quality of the equalized speech using ITU-T P.862 (PESQ) scores, which provides an estimate using a predicted mean opinion score (PMOS) ranging from 1 - 4.5 [25]. To allow comparison between the perceived quality of the reverberant microphone signals and the equalized speech signal, the difference in their PESQ scores is calculated as $\delta P$, where a positive $\delta P$ indicates an improvement in the equalized signal over the microphone signals. Our criteria for success is that the PESQ score should not be degraded in the second measure when the EDC is improved in the first measure. The third and final measure evaluates runtime using MATLAB's cputime function.

\subsection{Simulation 1: Subband RMCLS (RMCLS-SB)}

A 2-channel system was simulated using the image method [26, 27] with a room size of $6.4 \times 5 \times 3.6 \mathrm{~m}$, an inter-microphone distance of $0.1 \mathrm{~m}$, a distance of $2 \mathrm{~m}$ between the source and center of the microphone array and a reverberation time of $T_{60}=0.3 \mathrm{~s}$. The initial delay before the direct path in the AIRs was removed by fractional sampling such that $\tau=0$ and the channels were subsequently truncated to $L=2000$. Speech signals from the TIMIT database [28] were resampled to $f_{s}=8 \mathrm{kHz}$ and used as input. SIEs were artificially introduced by addition of white Gaussian noise to $\mathbf{h}$ to achieve an arbitrarily chosen illustrative level of NPM $=-33 \mathrm{~dB}$. For RMCLS, $L_{w}=0.05 f_{s}$ was used. For each simulation, 50 experiments were run with randomly varying locations of the source and microphone array while maintaining constant source-sensor distances to give spatially averaged results.

The averaged EDCs are given in Fig. 2 with the corresponding results for MINT included for reference. In the region of $t<0.05 \mathrm{~s}$, 
MINT-SB achieved improved suppression of $3 \mathrm{~dB}$ compared to full-band MINT whereas RMCLS-SB improved RMCLS by up to $15 \mathrm{~dB}$ for $t>0.03 \mathrm{~s}$. In the region of $t \geq 0.05 \mathrm{~s}$, both MINT and MINT-SB fail as they increase, rather than suppress, the energy in the reverberant tail whereas RMCLS-SB achieves suppression to within $1 \mathrm{~dB}$ of full-band RMCLS.

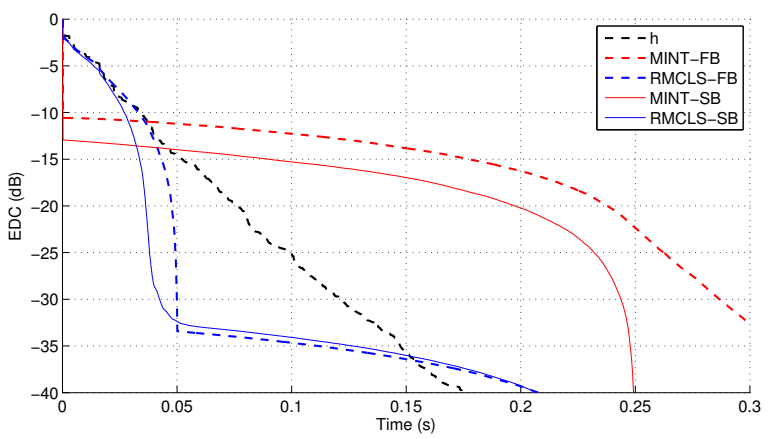

Fig. 2. Expt. 1 - Averaged EDCs with NPM $=-33 \mathrm{~dB}$.

The runtimes averaged over all experiments measured by MATLAB's cputime function was 311.53 for full-band RMCLS and 3.39 for RMCLS-SB, giving a reduction factor of 92 . The same reduction factor was achieved for MINT-SB over full-band MINT.

\subsection{Simulation 2: Gated Equalization in Subbands}

The value of $\mathrm{NPM}_{\mathrm{t}}$ was firstly determined for RMCLS-SB using the same acoustic system from Simulation 1 with 50 experiments run to obtain spatially averaged results. The averaged EDCs are given in Fig. 3, where it can be seen that $\mathrm{NPM}_{\mathrm{t}}=-12 \mathrm{~dB}$ as the EDC of $\mathbf{r}$ is greater than the EDC of $\mathbf{h}$ at $t=0.05 \mathrm{~s}$.

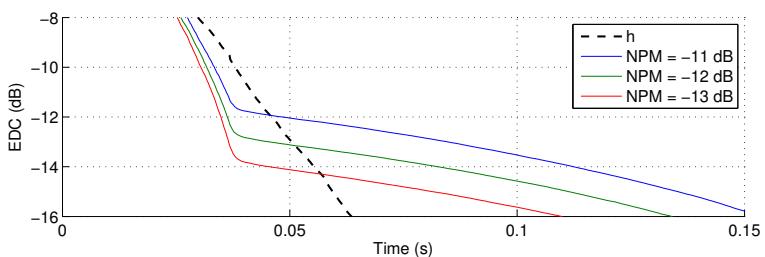

Fig. 3. Expt.2 - Averaged RMCLS EDCs with varying NPM.

\begin{tabular}{|c|c|c|c|}
\hline NPM & RMCLS-FB & RMCLS-SB & RMCLS-SB-G \\
\hline$-20 \mathrm{~dB}$ & 266.50 & 3.19 & 3.01 \\
$-12 \mathrm{~dB}$ & 335.40 & 3.51 & 1.98 \\
$-5 \mathrm{~dB}$ & 246.73 & 2.94 & 0.75 \\
\hline
\end{tabular}

Table 1. Expt. 2 - Averaged cputimes over a range of NPM values.

The performance of RMCLS-SB-G was then evaluated with NPM $=-5,-12,-20 \mathrm{~dB}$. It can be seen from the EDCs in Fig. 4 that when the SIEs are smaller than $\mathrm{NPM}_{\mathrm{t}}$, all algorithms under consideration were able to successfully suppress the reverberant tail. When the SIEs are greater than $\mathrm{NPM}_{t}$, no equalization was attempted by RMCLS-SB-G, successfully limiting the degradation that would have been introduced by the subband and full-band counterparts. At the threshold level, RMCLS-SB-G was able to achieve increased suppression rates beyond $t=0.05 \mathrm{~s}$. For example, at
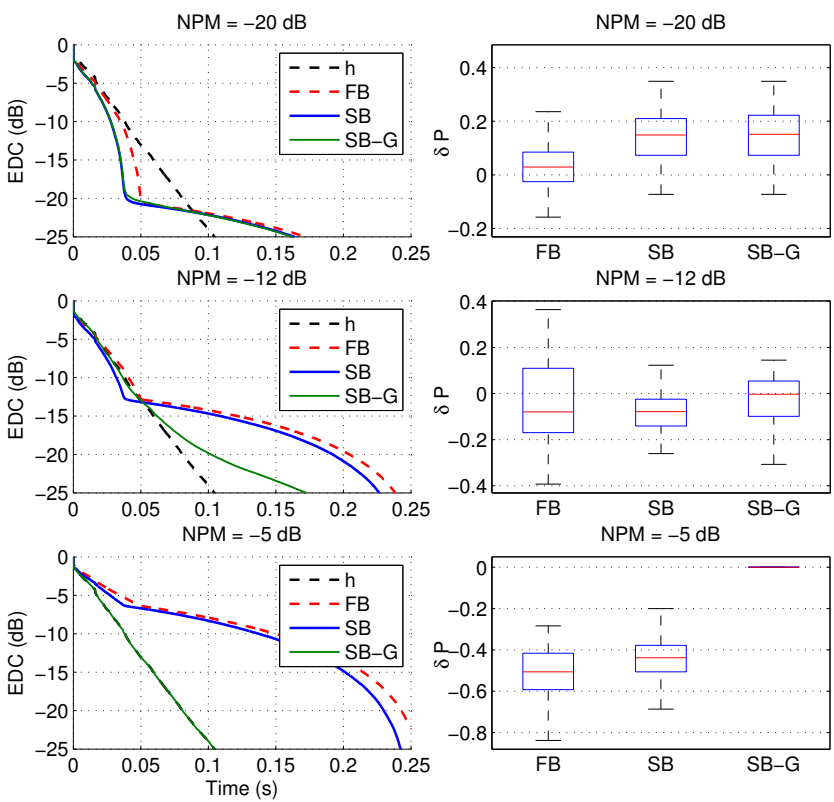

Fig. 4. Expt. 2 - Averaged RMCLS EDCs and $\delta P$ over a range of NPM values.

$t=0.1 \mathrm{~s}$, RMCLS-SB-G achieved an improvement of $5.2 \mathrm{~dB}$ over its subband counterpart.

The $\delta P$ scores are given in Fig. 4, where the line in the centre of each box indicates the median, the top and bottom of each box mark the interquartile range, and the dotted vertical lines represent the remaining data up to \pm 1.5 times the interquartile range. It can be seen that RMCLS-SB-G performs at least as well as RMCLS-SB and improves upon the performance of full-band RMCLS. Runtime was further reduced as shown in Table 1 especially in the cases where the level of SIE is larger than $\mathrm{NPM}_{t}$. This is as expected since equalization is not always performed in all subbands in that case.

\section{CONCLUSION}

We have presented a novel subband based equalizer, RMCLS-SB, and proposed gated subband equalization which provides greater control over equalization dependent on the expected levels of SIEs. Experimental results demonstrated that RMCLS-SB achieved improved reverberant tail suppression over its full-band counterpart as measured by EDCs, without deteriorating perceived quality of speech as measured by $\delta P$. As a result of subband processing, significant reduction in computational complexity and runtime of up to a factor of 92 was additionally achieved. Evaluation of gated subband equalization demonstrated that degradation in the EIR due to severe SIEs is avoided, therefore ensuring improved robustness of the dereverberation algorithms. Ongoing research includes extending the gated subband approach to applying varying levels of equalization.

\section{ACKNOWLEDGEMENT}

We thank Dr Nikolay Gaubitch of Delft University of Technology for providing MATLAB code relating to [16]. 


\section{REFERENCES}

[1] P. A. Naylor and N. D. Gaubitch, Eds., Speech Dereverberation, Springer, 2010.

[2] C. Avendano, J. Benesty, and D. R. Morgan, "A least squares component normalization approach to blind channel identification," in Proc. IEEE Intl. Conf. on Acoustics, Speech and Signal Processing (ICASSP), Mar. 1999, vol. 4, pp. 1797-1800.

[3] Y. Huang and J. Benesty, "Adaptive multi-channel least mean square and Newton algorithms for blind channel identification," Signal Processing, vol. 82, pp. 1127-1138, Aug. 2002.

[4] Y. Huang and J. Benesty, "A class of frequency-domain adaptive approaches to blind multichannel identification," IEEE Trans. Signal Process., vol. 51, no. 1, pp. 11-24, Jan. 2003.

[5] M. Miyoshi and Y. Kaneda, "Inverse filtering of room acoustics," IEEE Trans. Acoust., Speech, Signal Process., vol. 36, no. 2, pp. 145-152, Feb. 1988.

[6] I. Kodrasi, S. Goetze, and S. Doclo, "Increasing the robustness of acoustic multichannel equalization by means of regularization," in Proc. Intl. Workshop Acoust. Signal Enhancement (IWAENC), 2012, pp. 161-164.

[7] W. Zhang, E. A. P. Habets, and P. A. Naylor, "On the use of channel shortening in multichannel acoustic system equalization," in Proc. Intl. Workshop Acoust. Echo Noise Control (IWAENC), Tel Aviv, Israel, Aug. 2010.

[8] P. J. W. Melsa, R. C. Younce, and C. E. Rohrs, "Impulse response shortening for discrete multitone transceivers," IEEE Trans. Commun., vol. 44, pp. 1662-1672, 1996.

[9] N. Al-Dhahir, "FIR channel shortening equalizers for MIMO ISI channels," IEEE Trans. Commun., vol. 49, pp. 213-218, 2001.

[10] R. K. Martin, J. Balakrishnan, W. A. Sethares, and C. R. Johnson Jr., "A blind, adaptive TEQ for multicarrier systems," IEEE Signal Process. Lett., vol. 9, pp. 341-343, 2002.

[11] R. K. Martin, K. Vanbleu, M. Ding, G. Ysebaert, M. Milosevic, B. L. Evans, M. Moonen, and C. R. Johnson Jr., "Unification and evaluation of equalization structures and design algorithms for discrete multitone modulation systems," IEEE Trans. Signal Process., vol. 53, pp. 3880-3894, 2005.

[12] M. Kallinger and A. Mertins, "Impulse response shortening for acoustic listening room compensation," in Proc. Intl. Workshop Acoust. Echo Noise Control (IWAENC), Eindhoven, The Netherlands, 2005.

[13] M. Kallinger and A. Mertins, "Multi-channel room impulse response shaping - a study," in Proc. IEEE Int. Conf. Acoust., Speech, Signal Processing, 2006.

[14] A. Mertins, T. Mei, and M. Kallinger, "Room impulse response shortening/reshaping with infinity- and p-norm optimization," IEEE Trans. Audio, Speech, Lang. Process., vol. 18, no. 2, pp. 249-259, Feb. 2010.

[15] F. Lim and P. A. Naylor, "Relaxed multichannel least squares with constrained initial taps for multichannel dereverberation," in Proc. Intl. Workshop Acoust. Signal Enhancement (IWAENC), 2012.

[16] N. D. Gaubitch and P. A. Naylor, "Equalization of multichannel acoustic systems in oversampled subbands," IEEE Trans. Audio, Speech, Lang. Process., vol. 17, no. 6, pp. 1061-1070, Aug. 2009.
[17] G. Harikumar and Y. Bresler, "FIR perfect signal reconstruction from multiple convolutions: minimum deconvolver orders," IEEE Trans. Signal Process., vol. 46, pp. 215-218, 1998.

[18] T. Gerkmann and R. C. Hendriks, "Unbiased MMSE-based noise power estimation with low complexity and low tracking delay," IEEE Trans. Audio, Speech, Lang. Process., vol. 20, no. 4, pp. 1383 -1393, May 2012.

[19] M. R. P. Thomas, N. D. Gaubitch, E. A. P. Habets, and P. A. Naylor, "An insight into common filtering in noisy simo blind system identification," in Proc. IEEE Intl. Conf. on Acoustics, Speech and Signal Processing (ICASSP), Apr. 2012.

[20] K. Yamada, J. Wang, and F. Itakura, "Recovering of broad band reverberant speech signal by sub-band MINT method," in Proc. IEEE Intl. Conf. on Acoustics, Speech and Signal Processing (ICASSP), 1991, pp. 969-972.

[21] J. P. Reilly, M. Wilbur, M. Seibert, and N. Ahmadvand, "The complex subband decomposition and its application to the decimation of large adaptive filtering problems," IEEE Trans. Signal Process., vol. 50, no. 11, pp. 2730-2743, Nov. 2002.

[22] S. Weiss and R. W. Stewart, On adaptive filtering in oversampled subbands, Shaker Verlag, 1998.

[23] E. Anderson, Z. Bai, C. Bischof, S. Blackford, J. Demmel, J. Dongarra, J. Du Croz, A. Greenbaum, S. Hammarling, A. McKenney, and D. Sorensen, LAPACK Users' Guide, Society for Industrial and Applied Mathematics, Philadelphia, PA, third edition, 1999.

[24] G. H. Golub and C. F. van Loan, Matrix Computations, MD: John Hopkins University Press, Balimore, third edition, 1996.

[25] ITU-T, "Perceptual evaluation of speech quality (PESQ), an objective method for end-to-end speech quality assessment of narrowband telephone networks and speech codecs," Recommendation P.862, International Telecommunications Union (ITU-T), Feb. 2001.

[26] J. B. Allen and D. A. Berkley, "Image method for efficiently simulating small-room acoustics," J. Acoust. Soc. Am., vol. 65, no. 4, pp. 943-950, Apr. 1979.

[27] E. A. P. Habets, "Room impulse response (RIR) generator," http://home.tiscali.nl/ehabets/rirgenerator.html, May 2010.

[28] J. S. Garofolo, "Getting started with the DARPA TIMIT CD-ROM: An acoustic phonetic continuous speech database," Technical report, National Institute of Standards and Technology (NIST), Gaithersburg, Maryland, Dec. 1988. 